\title{
A Novel Anatomic Landmark to Target the Left Ventricle During Chest Compressions in Cardiac Arrest
}

Paul A. Olszynski ${ }^{1}$, Rhonda Bryce ${ }^{2}$, Qasim Hussain ${ }^{1}$, Stephanie Dunn ${ }^{3,4}$, Brandon Blondeau ${ }^{5}$, Paul Atkinson ${ }^{6,7}$, Robert Woods ${ }^{1}$

1. Emergency Medicine, University of Saskatchewan, Saskatoon, CAN 2. Clinical Research Support Unit, University of Saskatchewan, Saskatoon, CAN 3. Emergency Department, Royal University Hospital, Saskatoon, CAN 4. Faculty of Nursing, University of Regina, Saskatoon, CAN 5. School of Health Sciences, Saskatchewan Polytechnic, Saskatoon, CAN 6. Emergency Medicine, Saint John Regional Hospital, Saint John, CAN 7. Emergency Medicine, Dalhousie University, Halifax, CAN

Corresponding author: Paul A. Olszynski, p.olszynski@usask.ca

\section{Abstract \\ Background}

Resuscitation guidelines recommend that chest compressions be performed over the lower sternum. Current computed tomography and magnetic resonance imaging studies suggest that the current area of compression does not target the left ventricle (LV). Using transthoracic ultrasound, we sought to identify potential anatomic landmarks that would result in compressions over the LV in the majority of our study participants.

\section{Methodology}

We recruited 64 healthy men and women (over the age of 40) from the Simulated Patient Program at the University of Saskatchewan. Using ultrasound, we identified the LV and the associated surface anatomy in terms of intercostal space (ICS) and parasternal or mid-clavicular lines. We also collected biometric data including body mass index, chest circumference, and the corresponding inter-nipple line ICS.

\section{Results}

The LV was located along the left sternal border in 62 (96.9\%) participants. The most frequent LV location was along the left sternal border at the sixth ICS in 26 (40.6\%) participants, with $13(20.3 \%)$ at the fifth and 10 (15.6\%) participants at the seventh ICS. In two (3.1\%) participants, the LV was found along the midclavicular zone at the fifth ICS. The area from the fifth to seventh ICS on the left sternal border, typically covered by an adult palm centered at the sixth ICS, overlaid 49 of 64 (76.6\%, 95\% confidence interval [CI]: 64.3-86.2\%) identified LV locations. By comparison, centering the heel of the palm over the inter-nipple line at the left sternal border would cover the LV in 46 (71.9\%, 95\% CI: 59.2-82.4\%) participants.

Received 02/12/2021

Review began 02/14/2021 Review ended 02/22/2021 Published 03/02/2021

\section{() Copyright 2021}

Olszynski et al. This is an open access article distributed under the terms of the Creative Commons Attribution License CC-BY 4.0., which permits unrestricted use, distribution, and reproduction in any medium, provided the original author and source are credited.

\section{Conclusions}

A novel area of compression over the left sternal border at the inter-nipple line would result in compressions over the LV in nearly three-quarters of our study participants. Future research should investigate whether this proposed area of compression is applicable to a broader population including those with cardiac and thoracic disease.

Categories: Emergency Medicine

Keywords: anatomy, resuscitation, cardiac arrest, cpr, ultrasound

\section{Introduction}

Despite automated defibrillation and compression-focused resuscitation, out-of-hospital-cardiac-arrest survival remains $10 \%$ [1-3]. A mainstay of survival is effective chest compressions. According to the American Heart Association's cardiopulmonary resuscitation (CPR) guidelines, compressions should be performed over the lower half of the sternum [3]. During CPR, forward blood flow results from a combination of effects on the thoracic cavity and heart, generating flow through what are, respectively, known as the thoracic and cardiac pumps [4-6]. However, emerging research suggests that the current area of compression often results in outflow obstruction that may significantly limit or even compromise compression effect $[5,7$ 10].

A porcine study [11] demonstrated an increase in return of spontaneous circulation when compressions were performed directly over the center of the left ventricle (LV). The optimal compression zone was predetermined using transthoracic echocardiography (TTE) along the left sternal border by identifying 
the LV in its long axis. In recent years, use of transesophageal echocardiography (TEE) during human cardiac arrest has emerged as a means of guiding resuscitation and improving chest compressions. CPR quality can be improved by moving the area of compression according to visualized LV changes, including greatest LV compression [12,13], alleviation of outflow obstruction [14], and opening of the mitral valve with ventricular filling [15]. Evidence of increased cross-sectional area of the descending aorta immediately proximal to the point of maximal compression suggests that such compressions may have the added benefit of preferential cerebral blood flow [5]. Cardiac arrest guidelines for TEE outline the potential benefit of targeting LV compression to improve perfusion [16-18].

While TTE and TEE can be used to identify the LV and guide compressions, this is not currently a practical solution for out-of-hospital cardiac arrest. Using ultrasound, we sought to localize the LV and then correlate its location to external anatomic landmarks, thus identifying a novel area of compression that would overlie the LV in the majority of our study participants.

\section{Materials And Methods}

We obtained approval from the University of Saskatchewan's Research Ethics Board (Bio \#461) to conduct a pair of investigations exploring rapid localization of the LV through either sonographic [19] or anatomic landmark approaches. From January to April of 2019, we recruited a convenience sample of men and women over 40 years of age from the Health Sciences' Simulated Patient Program (University of Saskatchewan). We collected biometric data including weight, height, and chest circumference at the internipple line. Body mass index (BMI) was calculated as weight $(\mathrm{kg}) /$ height $^{2}(\mathrm{~m})$ and classified as normal $(<25)$, overweight (25-29.9), or obese ( $\geqslant 30)$.

Participants were placed in a supine position and ultrasound localization of the LV was performed by authors PO and RW using GE Venue 40 machines (General Electric, NY, USA). The ultrasound transducer was oriented along known echocardiographic windows including the parasternal long axis, apical four-chamber, and subcostal views. If a parasternal view of the heart was successfully obtained at the location, the space was deemed to be overlying either the long axis of the LV or aortic root (depending on which was the dominant structure in the view). If an apical four-chamber view was obtained, the space was deemed to be overlying the apex. If a subcostal view was obtained, the space was deemed to be overlying the inferior border of the heart (i.e., the right ventricle). Location of this position was plotted on a grid consisting of ICSs three to seven and two longitudinal zones: the left sternal border and mid-clavicular zones (see Figure 1). The nipple ICS was also documented in this supine position. All relevant images were saved and securely stored for subsequent review, as needed.

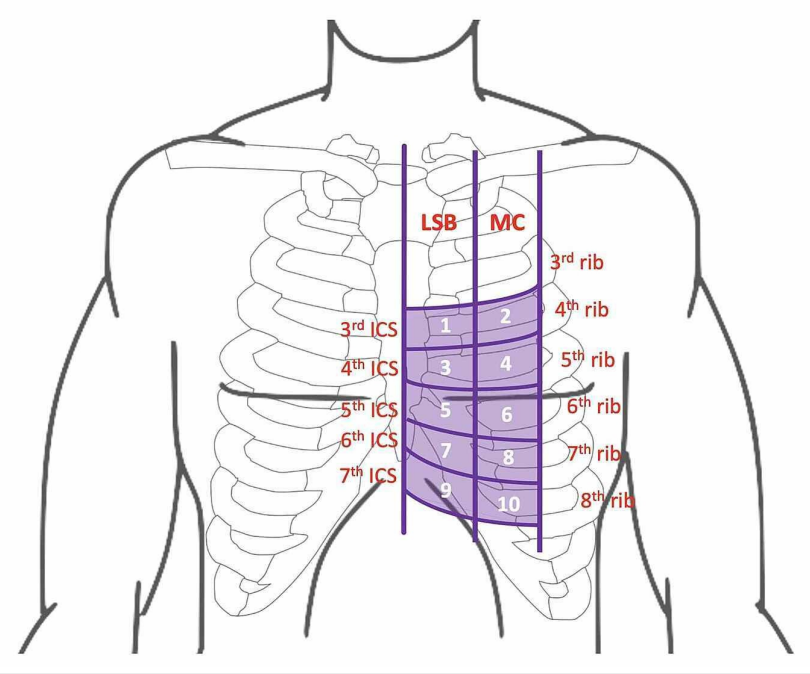

FIGURE 1: Ten-point interrogation grid.

LSB: left sternal border; MC: mid-clavicular; ICS: intercostal space

For the purpose of this study, we considered successful localization of the LV as an ICS where the long axis of the LV was the dominant structure in view (representing $>50 \%$ of horizontal axis). Ultrasound was also used to describe the participant's thorax including a scan for both pleural and pericardial effusions.

In clinical echocardiography, the LV is usually along the left sternal border between the third to fifth ICSs with the patient in left lateral decubitus [20,21]. These landmarks are of limited generalizability to the supine cardiac arrest patient undergoing CPR. Characteristics such as age, BMI, and certain medical 
conditions further contribute to the variability in terms of LV location [22]. While a detailed parasternal long axis view is not always achievable in the supine patient, it is nonetheless often sufficient to identify gross anatomy such as the LV. The location of the LV via the parasternal window (in terms of ICS and longitudinal line over the chest) represents our theorized optimal area of compression, as supported by porcine findings [11].

The most common means of compression in cardiac arrest is the heel of the human palm, measuring a mean width of 7-8 cm in females and 8-9 $\mathrm{cm}$ in males [23,24]. The average fourth to eighth ICSs (defined cranially by the inferior rib margin and caudally by the superior margin of the subsequent rib) show incremental widening from $1.3 \mathrm{~cm}( \pm 0.3 \mathrm{~cm})$ to $2.1 \mathrm{~cm}( \pm 0.4 \mathrm{~cm})$, respectively [25]. Combining ICSs and their associated rib widths, the average human palm spans approximately three ICSs. As such, we analyzed our findings in terms of not only the single best ICS overlying the LV but also as a range recognizing the width of the palm, including an ICS above and below the most common location of the LV. We submit that an area of compression where the $\mathrm{LV}$ is situated somewhere beneath the heel of the palm may be superior to compressions centered entirely away from the LV.

Descriptive statistics (means with standard deviations, frequencies with proportions) were calculated to characterize the demographic and anatomical features of participants. ICSs of the inter-nipple line and LV location were cross-tabulated to assess consistency; proportions agreeing and their 95\% confidence intervals (CIs) were determined. To account for the heel of the hand, agreement between anatomical landmarks and LV location was also assessed allowing for a margin of one ICS above and below the anatomical location. Agreement was assessed for the sample as a whole and by subgroups of sex and BMI. Exact CIs were determined for sex-specific and BMI category-specific proportions due to the relatively small number of participants in the subcategories. Proportions were compared between subgroups using the chisquare test. IBM SPSS Statistics for Windows, version 27 (IBM Corp, Armonk, NY, USA) was used for analysis.

\section{Results}

Demographic and anatomical details for participants are outlined in Table 1 . The LV was found along the left sternal border in 62 (96.9\%) participants. The most frequent location of the LV was the sixth ICS along the left sternal border in $26(40.6 \%)$ individuals, with $13(20.3 \%)$ at the fith and $10(15.6 \%)$ at the seventh ICS. The area from the fifth to seventh ICS on the left sternal border captured 49 of 64 or $76.6 \%$ (95\% CI: 64.386.2\%) of the identified LV locations. 


\section{Cureus}

\begin{tabular}{|c|c|c|c|}
\hline Characteristic & & Value & \\
\hline Age, y, mean (SD) & & 64.0 & (10.5) \\
\hline \multirow[t]{2}{*}{ Sex, n (\%) } & Male & 27 & (42.2) \\
\hline & Female & 37 & $(57.8)$ \\
\hline BMI, mean (SD) & & 29.0 & (6.4) \\
\hline \multirow[t]{3}{*}{ BMI, n (\%) } & Normal $(<25)$ & 17 & (26.6) \\
\hline & Overweight (25-29.9) & 23 & $(35.9)$ \\
\hline & Obese $(\geq 30)$ & 24 & (37.5) \\
\hline \multirow{3}{*}{ Chest circumference, $\mathrm{cm}$, mean (SD) } & Overall & 98.4 & (11.4) \\
\hline & Males & 99.9 & (9.2) \\
\hline & Females & 97.3 & (12.8) \\
\hline \multirow[t]{5}{*}{ Inter-nipple line intercostal space } & $4^{\text {th }}$ & 8 & (12.5) \\
\hline & $5^{\text {th }}$ & 19 & (29.7) \\
\hline & $6^{\text {th }}$ & 21 & (32.8) \\
\hline & $7^{\text {th }}$ & 14 & (21.9) \\
\hline & $8^{\text {th }}$ & 2 & (3.1) \\
\hline
\end{tabular}

TABLE 1: Demographic and anatomical characteristics of participants.

$\mathrm{BMI}=$ body mass index; $\mathrm{SD}=$ standard deviation; $y$ : years

The ICS of the inter-nipple line and LV were consistent among 21 (32.8\%, 95\% CI: 21.6-45.7\%) participants, all with the LV at the left sternal border. The region at the left sternal border covering one ICS above the inter-nipple line to one ICS below it overlaid the LV in 46 (71.9\%, 95\% CI: 59.2-82.4\%) participants. The degree of agreement between the level of the inter-nipple line and the LV are presented in Table 2.

\begin{tabular}{|c|c|c|c|c|c|c|}
\hline \multicolumn{2}{|c|}{ Location by echocardiography } & \multicolumn{5}{|c|}{ Corresponding inter-nipple line ICS, n } \\
\hline & n (\%) & $4^{\text {th }}$ & $5^{\text {th }}$ & $6^{\text {th }}$ & $7^{\text {th }}$ & $8^{\text {th }}$ \\
\hline ICS $3^{\text {rd }}$, LSB & $4(6.3)$ & 1 & 1 & 0 & 2 & 0 \\
\hline $4^{\text {th }}$, LSB & $9(14.1)$ & 1 & 4 & 3 & 1 & 0 \\
\hline $5^{\text {th }}$, LSB & $13(20.3)$ & 1 & 6 & 3 & 1 & 2 \\
\hline $5^{\text {th }}, \mathrm{MCL}$ & $2(3.1)$ & 1 & 0 & 1 & 0 & 0 \\
\hline $6^{\text {th }}$, LSB & $26(40.6)$ & 4 & 6 & 10 & 6 & 0 \\
\hline $7^{\text {th }}$, LSB & 10 (15.6) & 0 & 2 & 4 & 4 & 0 \\
\hline Total & 64 (100\%) & 8 & 19 & 21 & 14 & 2 \\
\hline
\end{tabular}

TABLE 2: Study findings for the frequency of LV position and ICSs.

LV = left ventricle; ICS = intercostal space; LSB = left sternal border; $M C L=$ mid-clavicular line 
the LV location by the inter-nipple line when stratified by sex or BMI, 15 of 37 (40.5\%, 95\% CI: $24.8-57.9 \%)$ females and six of 27 (22.2\%, 95\% CI: 8.6-42.3\%) males had exact matches between inter-nipple line ICS and LV ICS ( $\mathrm{p}=0.12) ; 27$ (73.0\%, 95\% CI: 55.9-86.2\%) females and 19 (70.4\%, 95\% CI: 49.8-86.2\%) males were shown to have the LV within one ICS of the inter-nipple line at the left sternal border $(\mathrm{p}=0.82)$. Among BMI subgroups, eight of 17 (47.1\%, 95\% CI: 23.0-72.2\%) normal BMI, four of 23 (17.4\%, 95\% CI: 5.0-38.8\%) overweight, and nine of 24 (37.5\%, 95\% CI: 18.8-59.4\%) obese participants demonstrated an exact match between the two locations ( $\mathrm{p}=0.12)$; 13 of 17 (76.5\%, 95\% CI: 50.1-93.2\%), 17 of 23 (73.9\%, 95\% CI: 51.689.8\%), and 16 of 24 (66.7\%, 95\% CI: 44.7-84.4\%) had their LV within one ICS above or below the nipple line at the left sternal border $(\mathrm{p}=0.76)$.

\begin{tabular}{|c|c|c|}
\hline LV location & n (\%) & $95 \% \mathrm{Cl}$ for proportion \\
\hline \multicolumn{3}{|c|}{ LV found at $6^{\text {th }}$ ICS, LSB, $n(\%)$} \\
\hline Yes & $26(40.6)$ & $28.5,53.6$ \\
\hline No & $38(59.4)$ & \\
\hline \multicolumn{3}{|c|}{ LV found within one ICS of the $6^{\text {th }}$ ICS (i.e., under palm coverage), LSB, $n(\%)$} \\
\hline Yes & $49(76.6)$ & $64.3,86.2$ \\
\hline No & $15(23.4)$ & \\
\hline \multicolumn{3}{|c|}{ LV found at nipple line, LSB, $n(\%)$} \\
\hline Yes & $21(32.8)$ & $21.6,45.7$ \\
\hline No & $43(67.2)$ & \\
\hline \multicolumn{3}{|c|}{ LV found within one ICS of nipple line (i.e., under palm coverage), LSB, n (\%) } \\
\hline Yes & $46(71.9)$ & $59.2,82.4$ \\
\hline No & $18(28.1)$ & \\
\hline
\end{tabular}

TABLE 3: Study findings describing LV position in relation to anatomic landmarks.

$\mathrm{LV}=$ left ventricle; ICS = intercostal space; $\mathrm{LSB}$ = left sternal border; $\mathrm{Cl}=$ confidence interval

\section{Discussion}

Our findings demonstrate that the LV is found to the left of the sternal border in a majority of supine adult participants. An area of compression over the left sternal border at either the sixth ICS or the inter-nipple line would result in compressions over the LV in the majority of our study participants. We report both of these anatomic locations because, despite evidence of improved LV localization at the sixth ICS, palpation of ICSs is challenging and has been found for chest tube insertion [26]. In contrast, the intersection of the left sternal border at the inter-nipple line presents a readily identifiable anatomic landmark for first responders. Differences between sex and BMI groups, though not statistically significant, warrant further consideration and investigation as obesity may limit generalizability.

Multiple radiological studies evaluating the location of critical cardiac structures have been published. In computed tomography (CT) and magnetic resonance imaging (MRI) studies, it has been demonstrated that the lower half of the sternum mostly compresses structures other than the LV including the ascending aorta, left atrium, right ventricle, aortic root, and proximal descending aorta [5,7-9].

A case series of five patients with TTE-guided LV compressions showed promising improvements in oxygen saturation and end-tidal $\mathrm{CO}_{2}$, but no improvement in survival. This may be attributed to the duration of standard compressions prior to LV localization and subsequent targeted LV compression [27]. The authors proposed integrating TTE at the start of CPR to optimize LV compression from the onset of resuscitation efforts. Similarly, TEE-guided compressions have not been shown to improve survival, possibly because this modality is often only implemented well into cardiac arrest care and the effects of targeted LV compression are blunted. Proposals to move the recommended hand position for chest compressions inferiorly and/or laterally to the left of the sternum have been previously made but met with reluctance due to concerns about possible impairment of the thoracic pump effect, as well as concern for injury to intra-abdominal organs $[8,10,21]$. 
Our study has several limitations. First, while we propose a novel area of compression overlying the LV, we present no data on the actual effects such a move would have on human patients during cardiac arrest in terms of hemodynamics, trauma to internal organs, survival, or other clinical outcomes. Our sample size was relatively small, and even though we did not screen our participants, the majority were generally healthy. Patients who suffer cardiac arrest likely have a higher prevalence of cardiac and thoracic pathology, most importantly cardiac dilation, which may result in lateral displacement of the LV and its axis. We used ultrasound to localize the heart as opposed to CT or MRI, and we did not perform blinded comparisons of location to ensure inter-rater reliability. While cardiac ultrasound is a user-dependent skill, both authors performing the scans have completed training in focused cardiac ultrasound consistent with the guidelines set forth by the Canadian Association of Emergency Physicians [28].

\section{Conclusions}

Utilizing ultrasound to map the position of the LV to the surface anatomy of our adult participants, we were able to identify an area of compression that may result in LV compression in most patients. Performing chest compressions over the left sternal border at the intersection of the inter-nipple line should be feasible for first responders and could optimize chest compression in cardiac arrest in a majority of patients. Further investigation is warranted to determine if the proposed area of LV compression is valid in a larger cohort of patients including those with cardiac and thoracic disease.

\section{Additional Information}

\section{Disclosures}

Human subjects: Consent was obtained or waived by all participants in this study. University of Saskatchewan issued approval Bio 461. This study was approved by the Research Ethics Board at the University of Saskatchewan. Animal subjects: All authors have confirmed that this study did not involve animal subjects or tissue. Conflicts of interest: In compliance with the ICMJE uniform disclosure form, all authors declare the following: Payment/services info: All authors have declared that no financial support was received from any organization for the submitted work. Financial relationships: Paul Atkinson declare(s) royalties from Oxford. Paul Atkinson declares royalties from Oxford. Paul Atkinson is the lead editor of Point of Care Ultrasound for Emergency Medicine and Resuscitation by Paul Atkinson, Justin Bowra, Tim Harris, Bob Jarman, and David Lewis. Oxford Clinical Imaging Guides; Oxford 2019. Other relationships: All authors have declared that there are no other relationships or activities that could appear to have influenced the submitted work.

\section{Acknowledgements}

The Ultrasound Localization to Resuscitate in Arrest (ULTRA) team would like to acknowledge Mr. Brandon Boyson and the staff at the Clinical Resource and Learning Centre (University of Saskatchewan) for their tremendous support and help with this study.

\section{References}

1. Sasson C, Rogers MA, Dahl J, Kellermann AL: Predictors of survival from out-of-hospital cardiac arrest: a systematic review and meta-analysis. Circ Cardiovasc Qual Outcomes. 2010, 3:63-81. 10.1161/CIRCOUTCOMES.109.889576

2. Nichol G, Leroux B, Wang H, et al.: Trial of continuous or interrupted chest compressions during CPR . N Engl J Med. 2015, 373:2203-2214. 10.1056/NEJMoa1509139

3. Kleinman ME, Brennan EE, Goldberger ZD, et al.: Part 5: adult basic life support and cardiopulmonary resuscitation quality: 2015 American Heart Association Guidelines update for cardiopulmonary resuscitation and emergency cardiovascular care. Circulation. 2015, 132:414-435. 10.1161/CIR.0000000000000259

4. Rudikoff MT, Maughan WL, Effron M, Freund P, Weisfeldt ML: Mechanisms of blood flow during cardiopulmonary resuscitation. Circulation. 1980, 61:345-352. 10.1161/01.CIR.61.2.345

5. Hwang SO, Lee KH, Cho JH, Yoon J, Choe KH: Changes of aortic dimensions as evidence of cardiac pump mechanism during cardiopulmonary resuscitation in humans. Resuscitation. 2001, 50:87-93. 10.1016/S03009572(01)00319-7

6. Georgiou M, Papathanassoglou E, Xanthos T: Systematic review of the mechanisms driving effective blood flow during adult CPR. Resuscitation. 2014, 85:1586-1593. 10.1016/j.resuscitation.2014.08.032

7. Pickard A, Darby M, Soar J: Radiological assessment of the adult chest: implications for chest compressions . Resuscitation. 2006, 71:387-390. 10.1016/j.resuscitation.2006.04.012

8. Cha KC, Kim YJ, Shin HJ, et al.: Optimal position for external chest compression during cardiopulmonary resuscitation: an analysis based on chest CT in patients resuscitated from cardiac arrest. Emerg Med J. 2013, 30:615-619. 10.1136/emermed-2012-201556

9. Shin J, Rhee JE, Kim K: Is the inter-nipple line the correct hand position for effective chest compression in adult cardiopulmonary resuscitation?. Resuscitation. 2007, 75:305-310. 10.1016/j.resuscitation.2007.05.003

10. Nestaas S, Stensæth KH, Rosseland V, Kramer-Johansen J: Radiological assessment of chest compression point and achievable compression depth in cardiac patients. Scand J Trauma Resusc Emerg Med. 2016, 24:54. 10.1186/s13049-016-0245-0

11. Anderson KL, Castaneda MG, Boudreau SM, Sharon DJ, Bebarta VS: Left ventricular compressions improve hemodynamics in a swine model of out-of-hospital cardiac arrest. Prehospital Emerg Care. 2017, 21:272280. 10.1080/10903127.2016.1241328 
12. Benato $\mathrm{P}$, Zanatta $\mathrm{M}$, Cianci V: Ultrasound guided chest compressions during cardiopulmonary resuscitation. Crit Ultrasound J. 2015, 7:32. 10.1186/2036-7902-7-S1-A32

13. Arntfield R, Pace J, Hewak M, Thompson D: Focused transesophageal echocardiography by emergency physicians is feasible and clinically influential: observational results from a novel ultrasound program. J Emerg Med. 2016, 50:286-294. 10.1016/j.jemermed.2015.09.018

14. Catena E, Ottolina D, Fossali T, et al.: Association between left ventricular outflow tract opening and successful resuscitation after cardiac arrest. Resuscitation. 2019, 138:8-14. 10.1016/j.resuscitation.2019.02.027

15. Kim H, Hwang SO, Lee CC, et al.: Direction of blood flow from the left ventricle during cardiopulmonary resuscitation in humans-its implications for mechanism of blood flow. Am Heart J. 2008, 156:1222. 10.1016/j.ahj.2008.09.003

16. Blaivas M: Transesophageal echocardiography during cardiopulmonary arrest in the emergency department . Resuscitation. 2008, 78:135-140. 10.1016/j.resuscitation.2008.02.021

17. Fair J, Mallin M, Mallemat H, et al.: Transesophageal echocardiography: guidelines for point-of-care applications in cardiac arrest resuscitation. Ann Emerg Med. 2018, 71:201-207. 10.1016/j.annemergmed.2017.09.003

18. Long B, Alerhand S, Maliel K, Koyfman A: Echocardiography in cardiac arrest: an emergency medicine review. Am J Emerg Med. 2018, 36:488-493. 10.1016/j.ajem.2017.12.031

19. Olszynski PA, Bryce R, Hussain Q, Dunn S, Blondeau B, Atkinson P, Woods R: Use of a simple ultrasound device to identify the optimal area of compression for out-of-hospital cardiac arrest. Cureus. 2021, 13:12785. 10.7759/cureus.12785

20. American College of Emergency Physicians: policy statement: emergency ultrasound imaging criteria compendium. (2006). Accessed: October 14, 2019: https://www.acep.org/patient-care/policystatements/Emergency-Ultrasound-Imaging-Criteria-Compendium/.

21. Lanctôt JF, Valois M: Echo guided life support. A-line Press, Montreal, Canada; 2016.

22. Hernandez C, Shuler K, Hannan H, Sonyika C, Likourezos A, Marshall J: C.A.U.S.E.: cardiac arrest ultrasound exam - a better approach to managing patients in primary non-arrhythmogenic cardiac arrest. Resuscitation. 2008, 76:198-206. 10.1016/j.resuscitation.2007.06.033

23. 2012 anthropometric survey of U.S. army personnel: methods and summary statistics . (2014). Accessed: October 14, 2019: https://ia802908.us.archive.org/3/items/DTIC_ADA611869/DTIC_ADA611869.pdf.

24. Choi H, Lee CC, Kim HJ, Singer AJ: Identifying the optimal hand placement site for chest compression by measuring hand width and sternal length in young adults. Am J Emerg Med. 2016, 34:407-411. 10.1016/j.ajem.2015.11.008

25. Dewhurst C, O'Neill S, O'Regan K, Maher M: Demonstration of the course of the posterior intercostal artery on CT angiography: relevance to interventional radiology procedures in the chest. Diagn Interv Radiol. 2011, 18:221-224. 10.4261/1305-3825.DIR.4366-11.1

26. Taylor LA, Vitto MJ, Joyce M, Tozer J, Evans DP: Ultrasound guided thoracostomy site identification in healthy volunteers. Crit Ultrasound J. 2018, 10:28. 10.1186/s13089-018-0108-1

27. Liu RB, Bogucki S, Marcolini EG, et al.: Guiding cardiopulmonary resuscitation with focused echocardiography: a report of five cases. Prehosp Emerg Care. 2020, 24:297-302. 10.1080/10903127.2019.1626955

28. Lewis D, Rang L, Kim D, et al.: Recommendations for the use of point-of-care ultrasound (POCUS) by emergency physicians in Canada. CJEM. 2019, 21:721-726. 10.1017/cem.2019.392 